\section{Recurrence Aortic Abdominal Dissection after Stent Grafting for Stanford Type B thoracic Dissection Case Report}

\section{Abstract}

Acute aortic dissection remains the most common aortic catastrophe currently. It is significant to be on the alert for the recurrent aortic dissection. A 62-yearold woman with hypertension was admitted to the Emergency department with sudden intense back pain, profuse sweating and with the feeling of impending death for 3 hours. The surgeon of Peking University Shougang hospital conducted TEVAR in the first day after hospitalization and the back pain had lessened after the surgeon. The fourth day after hospitalization, the patient had Intermittent back pain. The recurrent dissection entry was found on the bottom of the thoracic artery stent-graft. The surgeon of Peking University Shougang hospital conducted the endovascular covered stent-graft repair of the abdominal aorta.

Keywords: Type B thoracic dissection; Hypertension; Acute aortic dissection
Xiaoxiao Zhao ${ }^{1}$, Tianrun Lii ${ }^{2}$, Jinbo Liu ${ }^{1}$, Shaomei Feng ${ }^{1}$ and Hongyu Wang ${ }^{1 *}$

1 Department of Vascular Medicine, Peking University ShouGang Hospital, Beijing, China

2 The third Hospital of Peking University, Beijing, China

*Corresponding author: Hongyu Wang

” hongyuwang@188.com

Department of Vascular Medicine, Peking University ShouGang Hospital, Beijing, China.

Tel: 8610-57830226

Citation: Zhao X, Li T, Liu J, Feng S, Wang H (2018) Recurrence Aortic Abdominal Dissection after Stent Grafting for Stanford Type B thoracic Dissection Case Report. Biomark J. Vol.4 No.1:2

\section{Case}

A 62-year-old woman with hypertension was admitted to the Emergency Department with sudden intense back pain, profuse sweating and with the feeling of impending death for 3 hours. The pain was described as a constant and tearing feeling, which was not relieved by resting. She had a mild response to DanShen pill with sublingual administration in the pre-hospital setting. She was immediately sent for radiological examinations. Computed Tomography Angiography (CTA) findings indicated acute $B$ thoracic dissection (DeBakey III) with a wide range of hematomas which ranging from aortic arch to aorta descendent (Figure 1A). Imagine showed that the dissection entry was found on the aortic arch just distal to the left subclavian artery orifice (Figure 1B). The patient had 5 years of irregularly monitored hypertension with family history of hypertension and had an allergy to penicillin. She was transferred to the Vascular Medicine intensive care unit. Medical therapies to control pain and sustain hemodynamic stability were performed with continuous infusion of Bucinnazine, Urapidil Hydrochloride and nitroprusside. The patient was hemodynamically stable, with a blood pressure of $121 / 70 \mathrm{mmHg}$ and heart rate of 56 beats per minute. The electrocardiogram (ECG) showed signs of cardiac ischemia with the changes of $T$ waves. The left brachial ankle pulse wave velocity (ba-PWV) of the case is $2138 \mathrm{~cm} / \mathrm{s}$ and the right ba-PWV is $2018 \mathrm{~cm} / \mathrm{s}$.

The patient had uncontrolled hypertension, persistent pain. Therefore, emergency endovascular surgery was planned for repair of the complicated acute B thoracic dissection. The Thoracic Endovascular Aortic Repair (TEVAR) is considered a minimally invasive therapy, with lower operative morbidity and mortality rates than open surgical repair. The surgeon of Peking University Shougang hospital conducted TEVAR in the first day after hospitalization and the back pain had lessened after the surgeon. The final aortography revealed complete exclusion of the proximal entry site which maintained the blood supply to the abdominal aorta, and increased diameter of the true lumen. The Figure 2 showed the graft of the intraoperative cholangiography of the thoracic endovascular aortic repair. Computed tomography 

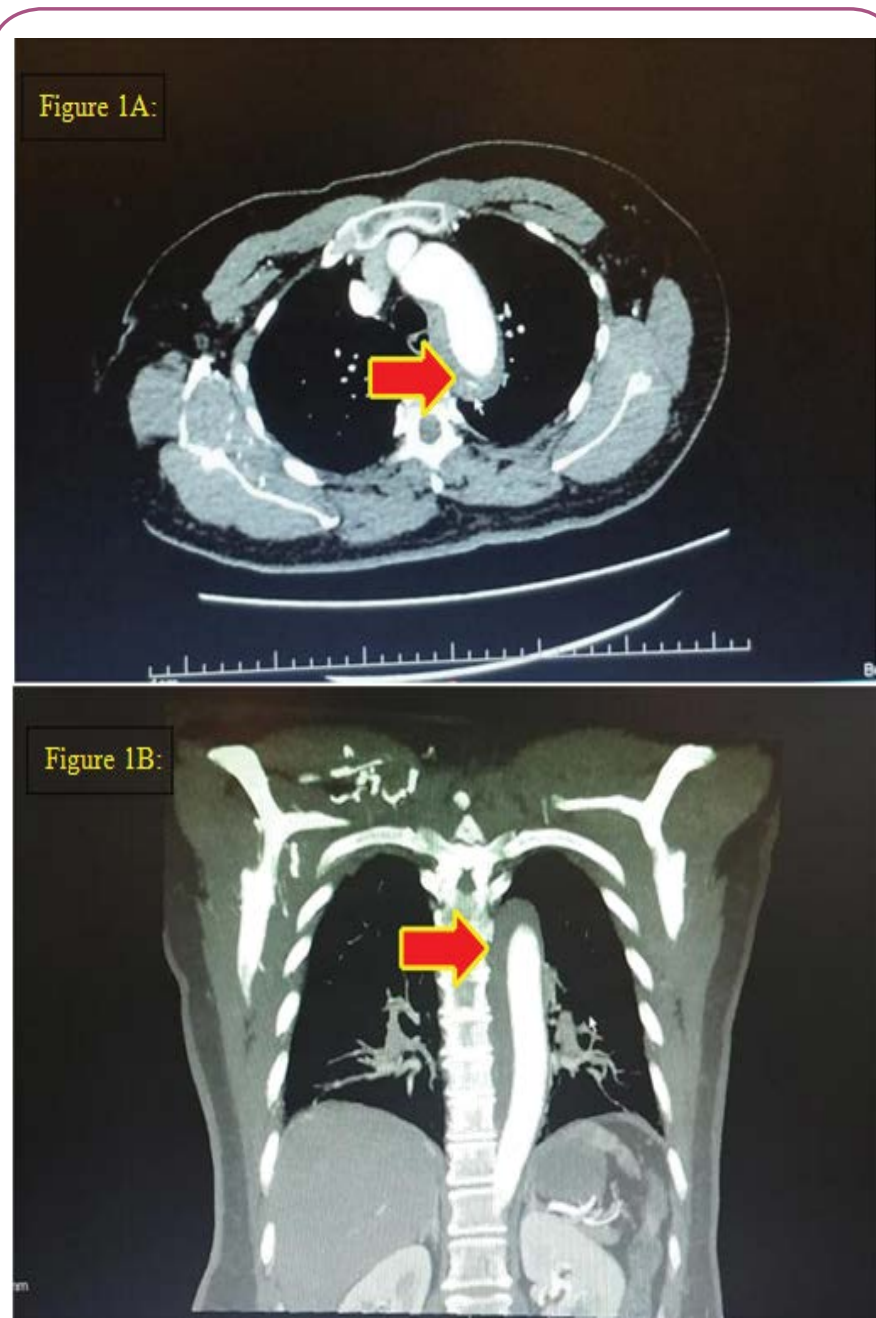

Figure 1 ( $\mathrm{A}, \mathrm{B})$ Computed tomography showed acute Stanford type B aortic dissection. The dissection entry was found on the aortic arch just distal to the left sub-clavian artery orifice. The initial computed tomography angiogram on the day of presentation demonstrating a descending thoracic aortic dissection with the true lumen identified by arrows.

2 days after the procedure showed that TEVAR was successful, resulting in expansion and patency of the true lumen with no endo-leak (Figure 3A and B). The fourth day after hospitalization, the patient had Intermittent back pain. Computed tomography angiography findings indicated recurrent abdominal aorta dissection with pleural effusion which eligible for the emergency interventional therapy. The recurrent dissection entry was found on the bottom of the thoracic artery stent-graft (Figure 4A and B). Comparing with the computed tomography imagine which conducted in the first day of the hospitalization (Figure 4C). The surgeon of Peking University Shougang hospital conducted the endovascular covered stent-graft repair of the abdominal aorta (Figure 5). The Figure 6 showed the graft of the intraoperative cholangiography of the abdominal endovascular aortic repair. However, the patient had Intermittent back pain once again



Figure 2 Showed the frame outlining of the aortic arch and its tributaries with an endovascular stent deployed immediately distal to the takeoff of the left subclavian artery in the intraprocedura I aortogram.

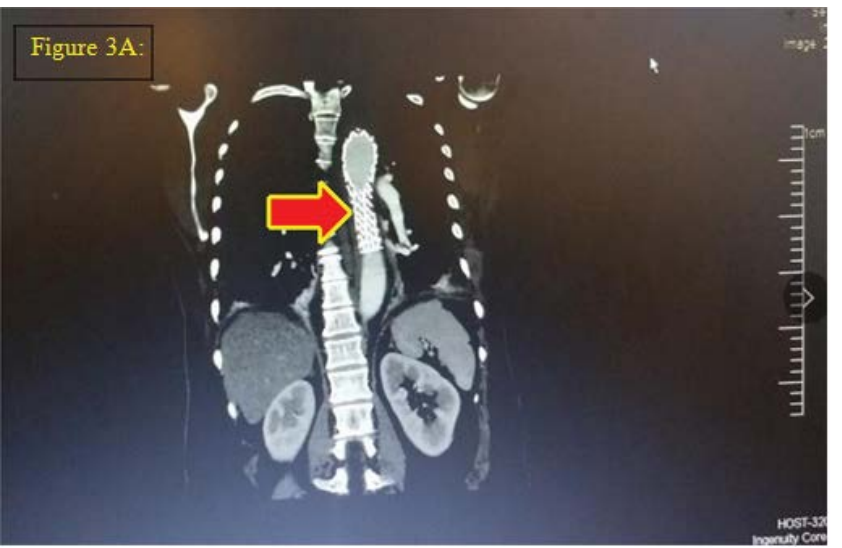

Figure 3A Computed tomography images performed at the 2 days after the procedure showed that TEVAR was successful. The endovascular graft can be visualized immediately.



in the sixth day after hospitalization. Repeated CTA findings indicated the absence of the recurrent aortic dissection (Figure 


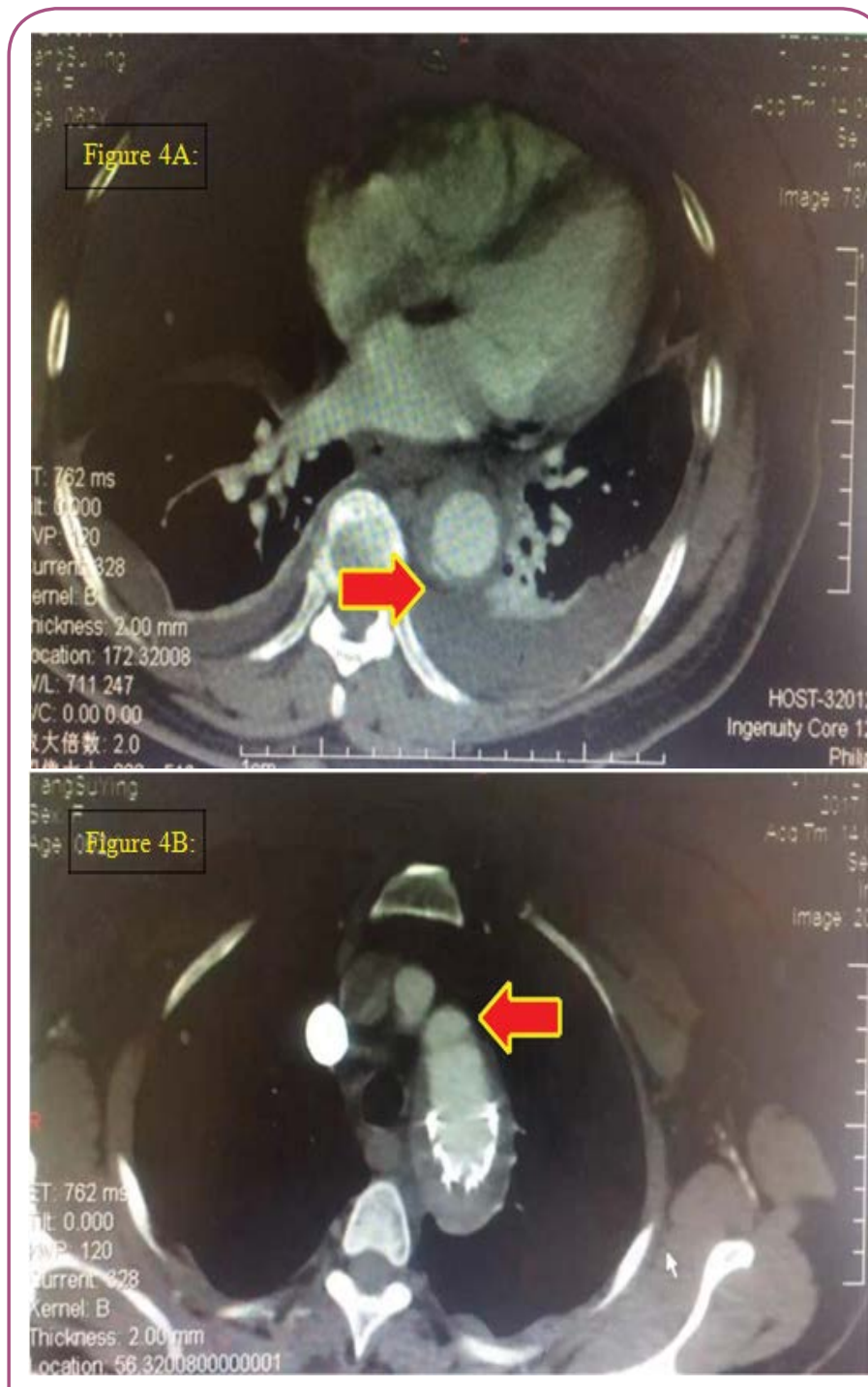

Figure $4 \quad(A, B)$ Computed tomography showed recurrent dissection was in the entry of recurrent abdominal aortic which just proximal to the previous stent graft after the patient complained of the recurrent back pain (red arrow).

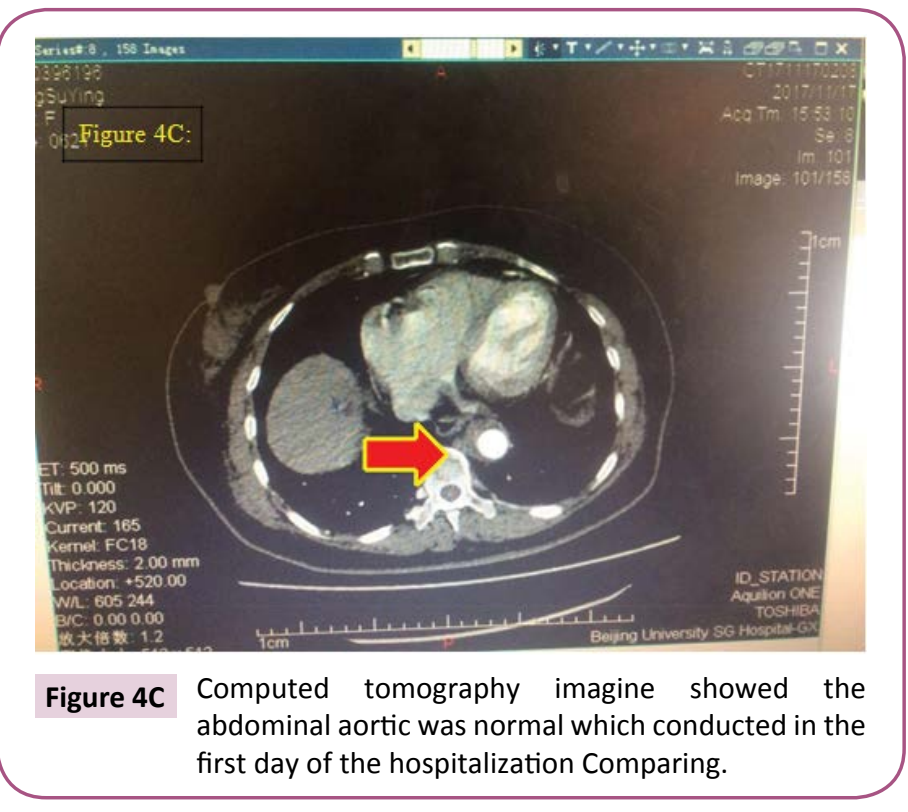




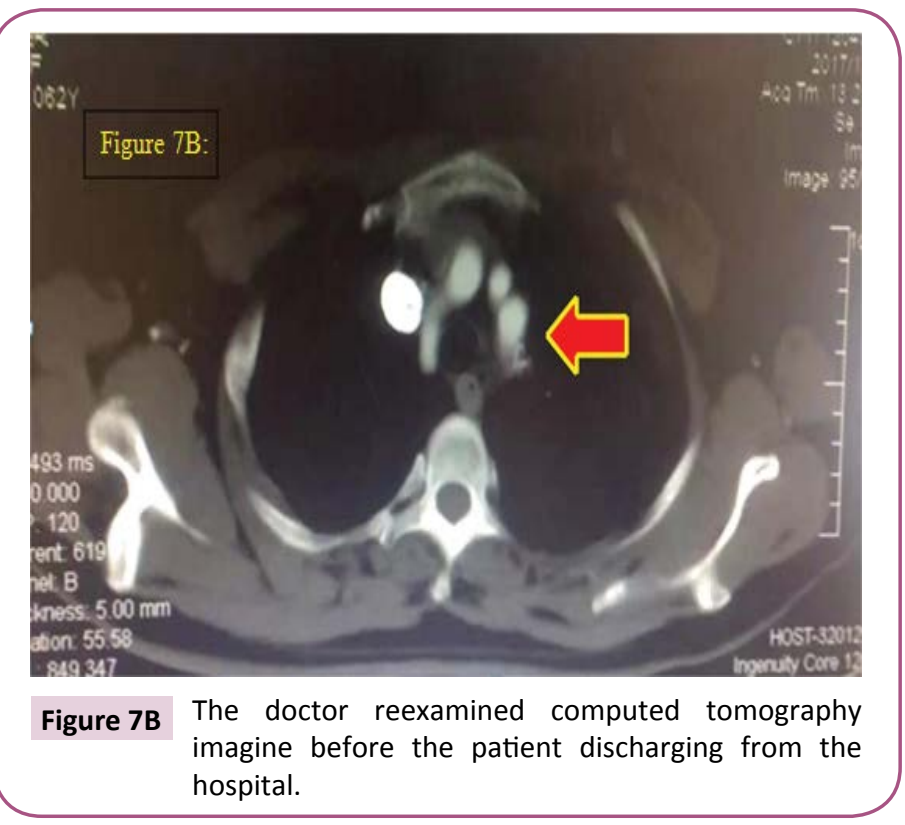

7A). The doctor reexamined computed tomography imagine before discharging from the hospital (Figure 7B).

\section{Discussion}

Acute aortic dissection remains the most common aortic catastrophe currently [1-3]. The type of the acute B aortic dissection was divided into complicated and uncomplicated Acute Type B Aortic Dissection (ATBAD). Complicated acute type $B$ aortic dissection was described as rupture, expansion of diameter on imaging during the admission, persistent pain, or clinical mal-perfusion leading to a deficit in cerebral, spinal, visceral, renal, or peripheral vascular territories at presentation or during initial hospitalization [4].

Patients with hypertension compounded by the stress and hemodynamic changes accompanying labor are predisposed to dissection as the aorta experiences increased shearing forces. To assess the influence of aortic stiffness on the extent of irreversible deformation and breaking stress of the human thoracic aorta, the team of Groenink M [5] conducted the research and found that Permanent deformation and rupture of the thoracic aorta following pressure overload are influenced by age, dispensability and collagen recruitment pressure. Because of the destruction of elastin leading to less strain-induced stiffening and predisposition

\section{References}

1 Clouse WD, Hallett JW Jr, Schaff HV, Spittell PC, Rowland CM, et al. (2004) Acute aortic dissection: population-based incidence compared with degenerative aortic aneurysm rupture. Mayo Clin Proc 79: 176-180.

2 White A, Broder J, Mando-Vandrick J, Wendell J, Crowe J (2013) Acute aortic emergencies, part 2: aortic dissections. Adv Emerg Nurs J 35: 28-52.

3 Safi HJ, Estrera AL (2004) Aortic dissection. Br J Surg 91: 523-525.

4 Rana OA, Harleen KS, Samuel SL, Mina LB, Varsha K, et al. (2015) to aortic dissection, the study of Nicholas R [6] suggest that hypertension is associated with a profound change in aortic wall mechanical properties possibly. Although it is remained that the computed tomography angiography is the top choice for imaging ATBAD, and magnetic resonance angiography is the second choice, these 2 modalities are static and present an arbitrary point in the cardiac cycle. Therefore, the dynamic natures of the dissection flap are unable to captured [7-9]. Recent publications acknowledge the importance of dynamic aortic evaluation in the treatment of aortic pathologies, especially aortic dissections [1013]. In our study, we measured the brachial ankle pulse wave velocity of the both and found that the values are higher than the normal indicating that the patient had the aortic artery stiffness which might distribute to the process of the aortic dissection.

The characteristics of the case are Refractory Pain and Hypertension. A multivariable logistic regression model from Professor Santi Trimarchi [14] confirmed that recurrent and/or refractory pain or refractory hypertension was a predictor of inhospital mortality particularly when managed medically and their observations suggest that aortic intervention may be indicated in the intermediate-risk group. The back pain of our case was occurred third times during the hospitalization. It is significant to be on the alert for the recurrent aortic dissection.

On the other hand, the leading to degeneration of the medial arterial layer, which may occur with greater frequency during pregnancy as hormonal fluctuations influence elastic fibril organization and vascular integrity is explored. Takayasu aortitis is an uncommon, idiopathic inflammatory disease which mostly affects the aorta and its main branches. Besides the nonspecific inflammatory symptoms, Takayasu aortitis also could be complicated by either ischemic symptoms attributed to stenotic lesions or by aortic wall dilation, disruption, and dissection [15]. However, the serum antibody was negative in this case. The follow-up management of the patient still is needed in the future.

\section{Conclusion}

We encountered a rare case of abdominal aortic dissection after TEVAR for complicated BAD, which were successfully repaired via emergency Stent Grafting. For patients complicated with type $B$ aortic dissection, TEVAR is a preferable and less invasive treatment option which could seal the proximal entry site to maintain true lumen blood flow effectively.

Outcomes of Patients With Acute Type B (DeBakey III) Aortic Dissection A 13-Year, Single-Center Experience. Circulation 132: 748-775.

5 Groenink M, Langerak SE, Vanbavel E, Vander Wall EE, Mulder BJ, et al. (1999) The influence of aging and aortic stiffness on permanent dilation and breaking stress of the thoracic descending aorta. Cardiovasc Res 43: 471-480.

6 Nicholas RG, Louise K, Antoine G, Alberto G, Sally B, et al. (2015) Altered Dependence of Aortic Pulse Wave Velocity on Transmural Pressure in Hypertension Revealing Structural Change in the Aortic Wall. Hypertension 65: 362-369. 
7 Van Herwaarden JA, Bartels LW, Muhs BE, Vincken KL, Lindeboom $M Y$, et al. (2006) Dynamic magnetic resonance angiography of the aneurysm neck: conformational changes during the cardiac cycle with possible consequences for endograft sizing and future design. J Vasc Surg 44: 22-28.

8 Laskowski I, Verhagen HJ, Gagne PJ, Moll FL, Muhs BE (2007) Current state of dynamic imaging in endovascular aortic aneurysm repair. J Endovasc Ther 14: 807-812.

9 Teutelink A, Muhs BE, Vincken KL, Bartels LW, Cornelissen SA, et al. (2007) Use of dynamic computed tomography to evaluate pre- and postoperative aortic changes in AAA patients undergoing endovascular aneurysm repair. J Endovasc Ther 14: 44-49.

10 Van Bogerijen GH, Van Herwaarden JA, Conti M, Auricchio F, Rampoldi V, et al. (2014) Importance of dynamic aortic evaluation in planning TEVAR. Ann Cardiothorac Surg 3: 300-306.

11 Clough RE, Zymvragoudakis VE, Biasi L, Taylor PR (2014) Usefulness of new imaging methods for assessment of type B aortic dissection. Ann Cardiothorac Surg 3: 314-318.

12 Kinner S, Eggebrecht H, Maderwald S, Barkhausen J, Ladd SC, et al. (2015) Dynamic MR angiography in acute aortic dissection. J Magn Reson Imaging 42: 505-514.

13 Clough RE, Waltham M, Giese D, Taylor PR, Schaeffter T (2012) A new imaging method for assessment of aortic dissection using fourdimensional phase contrast magnetic resonance imaging. J Vasc Surg 55: 914-923.

14 Trimarchi S, Kim AE, Christoph AN, Reed EP, Frederik HW, et al. Importance of Refractory Pain and Hypertension in Acute Type B Aortic Dissection Insights from the International Registry of Acute Aortic.

15 Isobe M (2013) Takayasu arteritis revisited: current diagnosis and treatment. Int J Cardiol 168: 3-10. 